\title{
Invasive micropapillary carcinoma of mixed breast cancer metastasizing to the cervical region and thyroid: report of a rare case
}

\author{
Yupei Yu ${ }^{1}$, Ruifeng Wang ${ }^{1}$, Junqi Deng $^{2}$, Jiayu Zhou ${ }^{1,2}$, Haiyan Zhou ${ }^{1,2}$, Junpu Wang $^{1,2}$ \\ ${ }^{1}$ Department of Pathology, Xiangya Hospital, Central South University, Changsha, China; ${ }^{2}$ Department of Pathology, School of Basic Medicine, \\ Central South University, Changsha, China \\ Correspondence to: Junpu Wang; Haiyan Zhou. Department of Pathology, Xiangya Hospital, Central South University, 87 Xiangya Road, Kaifu \\ District, Changsha 410008, China. Email: wang-jp2013@csu.edu.cn; yanhaizhou78@163.com.
}

\begin{abstract}
While the incidence of metastatic breast cancer tumors in the thyroid gland is extremely rare, invasive micropapillary carcinoma (IMPC) of the breast metastasizing to the thyroid gland is even rarer. There are no known reported cases in which both invasive ductal carcinoma-no special type (IDC-NST) and IMPC existed in the breast tissue and where only the latter metastasized into the thyroid gland. This report details the case of a 59-year-old Chinese woman who was first diagnosed with grade 2 IDC-NST of the breast with involvement of 6 axillary lymph nodes in 2015. However, 5 years later, blood tests revealed increased tumor markers. Imaging revealed multiple solid nodules in both sides of the thyroid gland. Total thyroidectomy and bilateral cervical lymph node dissection were performed; the findings showed both IDCNST and IMPC in the lymph nodes, but only the latter in the thyroid gland. Immunohistochemically, these tumor cells were positive for estrogen receptor (ER), progesterone receptor (PR), GATA binding protein 3 (GATA3), and mammaglobin, but negative for thyroglobulin (TG) and thyroid transcription factor-1 (TTF-1). Ultimately, the patient was diagnosed as having mixed breast cancer with metastatic IMPC in the thyroid gland, and chemotherapy was continued. Apart from the possibility of IDC-NST in breast tissue metastasizing to the thyroid, metastasis of IMPC of the breast must also be taken into consideration, especially when the patient has a history of breast cancer and thyroid nodules. Accurate diagnosis of metastatic breast carcinoma is vital for precise treatment and can improve the prognosis of patients.
\end{abstract}

Keywords: Tumor metastasis; mixed breast cancer; invasive micropapillary carcinoma (IMPC); thyroid; case report

Submitted Jul 29, 2021. Accepted for publication Oct 20, 2021.

doi: $10.21037 / g s-21-506$

View this article at: https://dx.doi.org/10.21037/gs-21-506

\section{Introduction}

Primary breast and thyroid tumors are a common invasive disease for women worldwide, and an increase in the incidence of both has been well documented in recent years $(1,2)$. However, metastatic breast cancer presenting in the thyroid gland is recognized as an uncommon phenomenon (3). The typical remote sites for secondary relapse of breast neoplasm are the bones, liver, brain, and lungs, but not the thyroid (4).

The most common form of invasive breast cancer, invasive ductal carcinoma (IDC), was recently divided into invasive ductal carcinoma-no special type (IDC-NST) and other specific subtypes (5). Invasive micropapillary carcinoma (IMPC) is a subtype of IDC that accounts for approximately $7 \%$ of all breast cancer types. IMPCs are characterized by small, hollow, or morule-like clusters of neoplastic cells without fibrovascular cores, and are situated in distinct empty spaces $(6,7)$. Local lymph nodes and distant organs are commonly involved in IMPC of the breast, and this involvement typically results in poor prognosis. This report presents a peculiar case in which the 

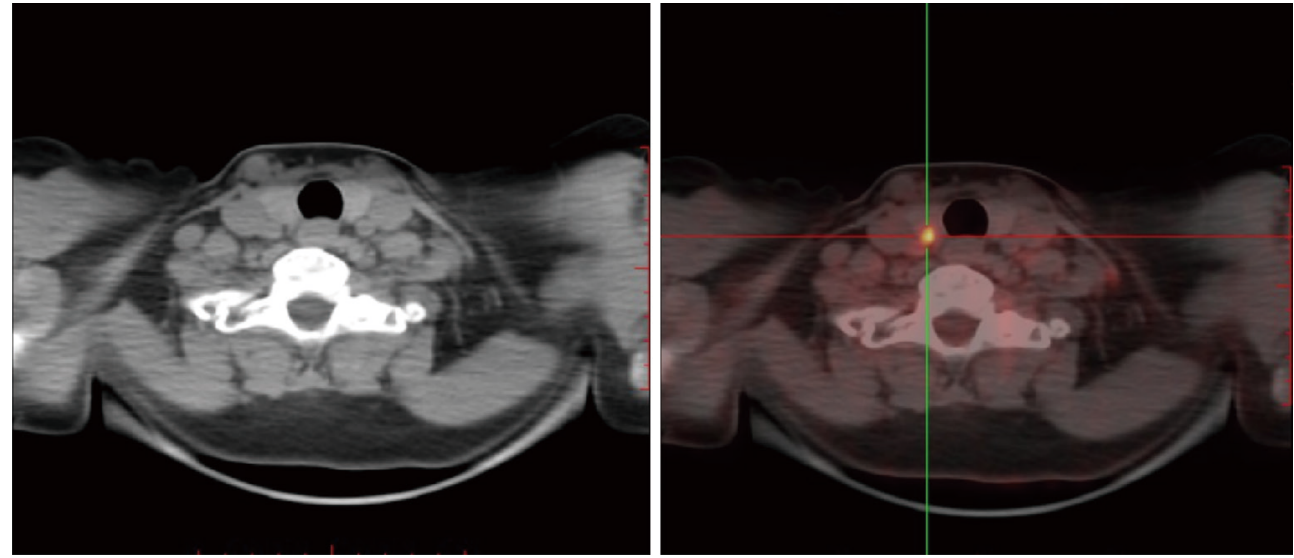

Figure 1 PET-CT revealed increased focal FDG uptake in the thyroid gland mass.

cervical region including thyroid gland was invaded much more easily by IMPC than by IDC-NST of the breast. The following case is presented in accordance with the CARE reporting checklist (available at https://dx.doi.org/10.21037/ gs-21-506).

\section{Case presentation}

In 2015, a 59-year-old woman presented to the hospital complaining of a lump in her right breast, and thorough clinical examinations were undertaken. Bilateral mammography showed an irregular, hyperdense mass in the right retro areolar region (see Figure S1A). Ultrasonography revealed a $1.7 \mathrm{~cm} \times 1.3 \mathrm{~cm}$ hypoechoic irregular mass with no blood flow at 6 o'clock direction of the right breast and enlargement of multiple axillary lymph nodes (see Figure S1B). Core needle biopsy revealed that the cancer was IDC-NST, grade 2 (according to the Nottingham grading system). A right modified radical mastectomy was performed, and postoperative pathology confirmed both the histopathological tumor classification and the grade were same as the previous diagnosis, with involvement of 6 axillary lymph nodes. Immunohistochemical study showed the following: estrogen receptor (ER), 90\%+; progesterone receptor (PR), 100\%+; and human epidermal growth factor receptor 2 (HER2), 2+. Fluorescence in situ hybridization (FISH) results were negative for HER2 gene amplification, so the molecular subtype was identified as luminal $\mathrm{A}$. The patient subsequently received adjuvant chemotherapy with 4 cycles of pirarubicin and cyclophosphamide regimens followed by docetaxel for 4 cycles, which was followed by toremifene therapy for 3 years. Periodic imaging and biochemical examinations were performed every 3 months.

In 2018, the patient had a relapse in her right chest wall, and the mass was removed. She was started on additional radiation therapy and letrozole instead of toremifene to treat the breast cancer. After 2 years, blood tests revealed an increased carcinoembryonic antigen (CEA) level of $12.2 \mathrm{ng} / \mathrm{mL}$ (reference range, $<5.2 \mathrm{ng} / \mathrm{mL}$ ), CA199 of $42.17 \mathrm{U} / \mathrm{mL}$, and CA125 of $55.99 \mathrm{U} / \mathrm{mL}$. A positron emission tomography-computed tomography (PET-CT) scan was performed and showed elevated uptake in the thyroid gland mass [standard uptake volume (SUV) max: 6.3; Figure 1], multiple cervical lymph nodes on both sides (SUV max: 4.9), and mediastinal lymph nodes (SUV max: 7.5). Ultrasonography of the neck revealed that multiple solid nodules were present on both sides of the thyroid gland, with microcalcifications in the right lobe. Fineneedle aspiration (FNA) revealed the thyroid nodules were malignant. Total thyroidectomy and bilateral cervical lymph node dissection were performed. The HE-stained sections of thyroid gland and the history of breast cancer showed the thyroid gland nodules were inclined to metastatic tumors from the breast.

Wax blocks and HE-stained sections of the thyroid gland were sent to the pathology outpatient department for a definitive diagnosis. Histopathological examination revealed invasive tumor cells with lympho-vascular invasion (LVI) in both thyroid lobes in both thyroid lobes formed by pseudopapillary fragments composed of cell clusters with large, granular cytoplasm lacking fibrovascular cores, and floating in clear, empty spaces (Figure 2A). Immunohistochemical study to clarify the origin of the tumor (Figure 2B-2D) revealed the following: 


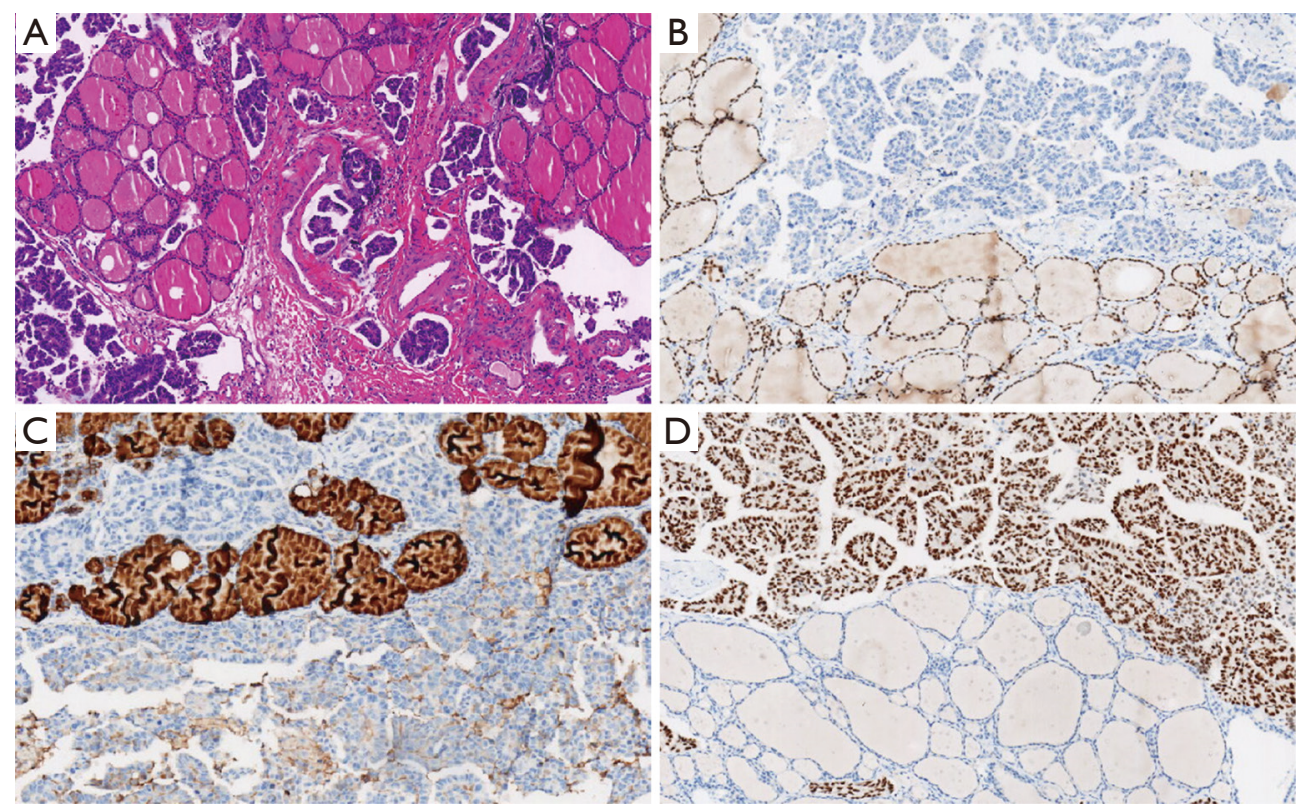

Figure 2 Histological and immunohistochemical results of the tumor. (A) Metastatic invasive micropapillary carcinoma in the thyroid gland (HE staining, magnification $\times 40$ ); (B) thyroid transcription factor-1 (TTF-1) negativity in the metastatic tumor area; (C) thyroglobulin (TG) negativity in breast cancer metastasis cells; (D) GATA-3 positivity in breast cancer metastasis cells. (B-D: immunohistochemistry staining, magnification $\times 40$ ).

thyroglobulin (TG)(-), thyroid transcription factor-1 (TTF-1)(-), GCDFP-15(-), ER (3+, 6\%), PR (1+, 30\%), HER2(3+), GATA3(3+), and mammaglobin (1+). As HER2 amplification was detected, the metastatic breast cancer was classified as HER2-positive breast cancer. A further diagnosis of metastatic micropapillary carcinoma of breast to the thyroid was made.

To determine further treatment, paraffin sections from a mastectomy specimen and axillary lymph node were reviewed. Histopathology showed both IDC-NST and IMPC, indicating that the patient was misdiagnosed in the first instance (Figure 3A,3B). Immunohistochemical staining revealed that characteristics of epithelial membrane antigen (EMA) expression exhibited reversed polarity, and E-cadherin was positive in most of the cell membrane for the tumor studies, which were distinct only in IMPC tumors (Figure 3C,3D). Ultimately the patient was diagnosed with mixed breast cancer (IDC-NST and IMPC) with metastatic thyroid tumor. The patient was then given chemotherapy combined with targeted therapies, followed by nanoparticle albumin-bound paclitaxel, trastuzumab, and pertuzumab in January 2021. To date, patient monitoring using imaging and biochemical testing has indicated that the disease remains stable. All procedures performed in studies involving human participants were in accordance with the ethical standards of the institutional and/or national research committee(s). The study was conducted in accordance with the Declaration of Helsinki (as revised in 2013). Written informed consent was obtained from the patient for publication of this case report and accompanying images. A copy of the written consent is available for review by the editorial office of this journal.

\section{Discussion}

Metastatic disease of the thyroid gland serves as recipient to non-thyroid sites metastasis, which is an uncommon phenomenon and has been attributed to the fast flow of arterial blood and the abundant iodine and oxygen content found in the highly vascularized thyroid gland $(8,9)$. Breast cancer has a high likelihood of metastasizing to distant organs, such as the bones, liver, brain, and lungs, and the 5 -year overall survival rate of patients with metastasis is much lower than that in patients without metastasis (10). However, the incidence rate of breast cancer metastasizing to the thyroid gland is low, and very few cases have been reported to date (11-14). Notably, there is only one other reported case of IMPC of the breast metastasizing to the thyroid gland (11). 


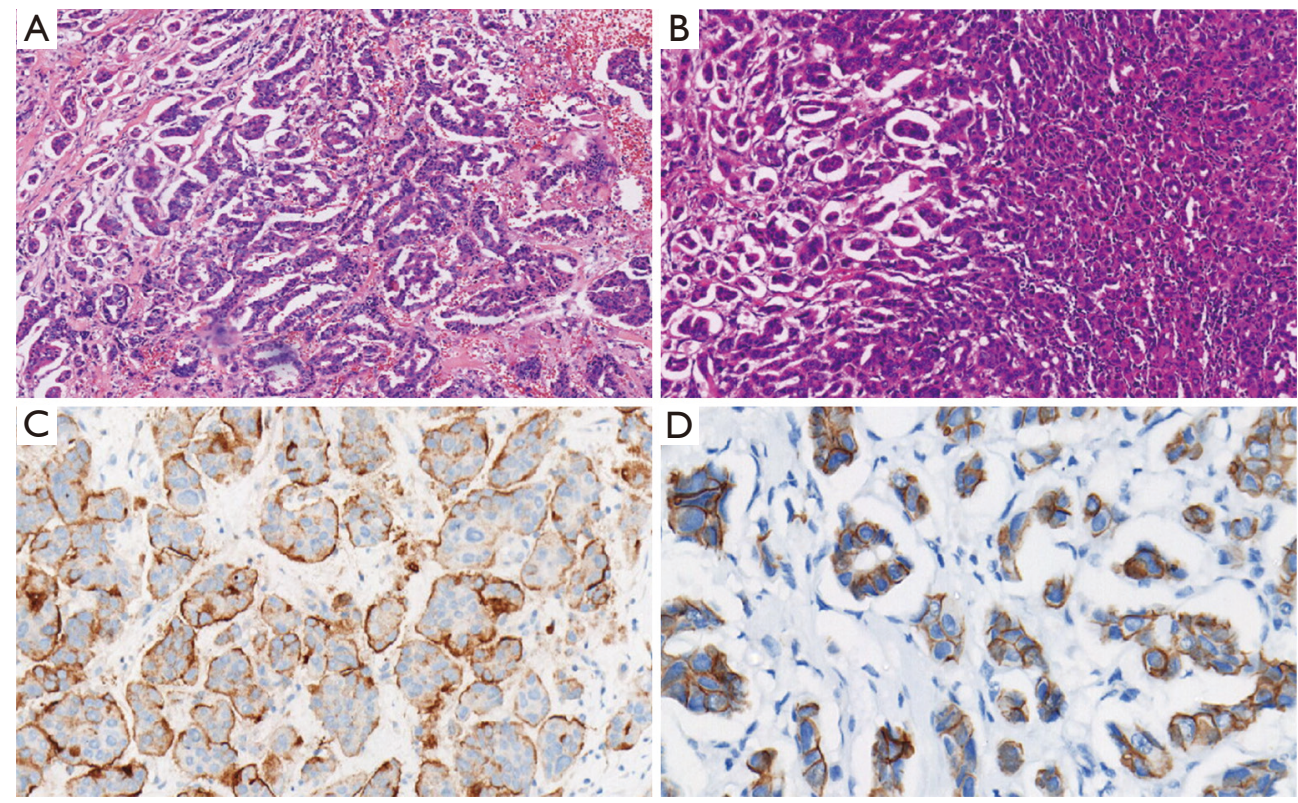

Figure 3 Histopathological features of the mixed breast cancer. (A,B) Both invasive micropapillary carcinoma (IMPC) and invasive ductal carcinoma in the breast and axillary lymph node (A and B: HE staining, magnification $\times 40)$; (C) characteristics of epithelial membrane antigen (EMA) immunostaining showed reversed polarity of IMPC cells in the axillary lymph node (magnification $\times 100)$; (D) E-cadherin staining was positive in most of the cell membrane of tumor cells in the axillary lymph node (magnification $\times 200$ ).

Here, we present a patient with distinct metastatic breast cancer that had 2 components, IDC-NST and IMPC. The patient had a metastasis of IDC-NST and IMPC to the lymph nodes, but 5 years later, only the IMPC metastasized to both lobes of the thyroid. Although immunohistochemistry can differentiate between IMPC and IDC-NST, misdiagnosis is common, especially when both subtypes are present. In this case, mixed components of breast cancer metastasized to the axillary lymph nodes, while only IMPC was discovered in the cervical region, including the thyroid gland. This biological behavior can more likely be attributed to lymphatic metastasis, and, in fact, reports have confirmed that IMPC has a higher proclivity to lymph vascular invasion and lymph node metastasis as compared to IDC, which could explain these findings (15). Our results suggest that IMPC metastasizes into distant organs much more easily than do other subtypes of invasive breast cancer. In addition, the molecular subtype of this case should be considered. The cancer was identified as luminal A type (ER+ and/or PR+, and HER2-) after breast surgery, but 5 years later, the metastatic carcinoma was HER2+, which may be closely related to the biological behavior observed in this case.

Clinical data have shown that IMPC is easily confused for other types of invasive neoplasms, IDC in particular, and that pure-component IMPC (more than $75 \%$ ) is extremely rare (16). Reports have confirmed that IDC + IMPC patients present with a higher proportion of vascular invasion, and that metastasis and death occur more often in these patients compared to pure IDC and IDC + ductal carcinoma in situ (DCIS) patients (17).

In conclusion, apart from the possibility of metastatic IDC-NST to the thyroid, pathologists should take metastasis of IMPC of the breast into consideration, especially when the patient has a history of breast cancer and thyroid nodules. It is very important to make an accurate diagnosis of IMPC in primary breast cancers to promote therapy and improve prognosis of patients. Specific morphology, immunohistochemical characteristics are all helpful for diagnosis when IMPC of the breast has metastasized to the thyroid. In addition, HER2 FISH gene detection plays an important role and the HER2 positive patient is beneficial from trastuzumab and pertuzumab.

\section{Acknowledgments}

Funding: None. 


\section{Footnote}

Reporting Checklist: The authors have completed the CARE reporting checklist. Available at https://dx.doi. org/10.21037/gs-21-506

Peer Review File: Available at https://dx.doi.org/10.21037/ gs-21-506

Conflicts of Interest: All authors have completed the ICMJE uniform disclosure form (available at https://dx.doi. org/10.21037/gs-21-506). The authors have no conflicts of interest to declare.

Ethical Statement: The authors are accountable for all aspects of the work in ensuring that questions related to the accuracy or integrity of any part of the work are appropriately investigated and resolved. All procedures performed in studies involving human participants were in accordance with the ethical standards of the institutional and/or national research committee(s). The study was conducted in accordance with the Declaration of Helsinki (as revised in 2013). Written informed consent was obtained from the patient for publication of this case report and accompanying images. A copy of the written consent is available for review by the editorial office of this journal.

Open Access Statement: This is an Open Access article distributed in accordance with the Creative Commons Attribution-NonCommercial-NoDerivs 4.0 International License (CC BY-NC-ND 4.0), which permits the noncommercial replication and distribution of the article with the strict proviso that no changes or edits are made and the original work is properly cited (including links to both the formal publication through the relevant DOI and the license). See: https://creativecommons.org/licenses/by-nc-nd/4.0/.

\section{References}

1. Roman BR, Randolph GW, Kamani D. Conventional Thyroidectomy in the Treatment of Primary Thyroid Cancer. Endocrinol Metab Clin North Am 2019;48:125-41.

2. Ferlay J, Colombet M, Soerjomataram I, et al. Cancer statistics for the year 2020: An overview. Int J Cancer 2021. [Epub ahead of print].

3. Tan W, Tao L, Zhou Z, et al. Tumor-to-tumor metastasis: a rare case of breast carcinoma metastasizing to a pheochromocytoma, and a literature review. Diagn Pathol
2019;14:46.

4. Kennecke H, Yerushalmi R, Woods R, et al. Metastatic behavior of breast cancer subtypes. J Clin Oncol 2010;28:3271-7.

5. Jenkins S, Kachur ME, Rechache K, et al. Rare Breast Cancer Subtypes. Curr Oncol Rep 2021;23:54.

6. Shi WB, Yang LJ, Hu X, et al. Clinico-pathological features and prognosis of invasive micropapillary carcinoma compared to invasive ductal carcinoma: a population-based study from China. PLoS One 2014;9:e101390.

7. Song $\mathrm{Y}$, Sun $\mathrm{H}, \mathrm{Wu} \mathrm{K}$, et al. sLex expression in invasive micropapillary breast carcinoma is associated with poor prognosis and can be combined with MUC1/EMA as a supplementary diagnostic indicator. Cancer Biol Med 2021. [Epub ahead of print].

8. Nixon IJ, Coca-Pelaz A, Kaleva AI, et al. Metastasis to the Thyroid Gland: A Critical Review. Ann Surg Oncol 2017;24:1533-9.

9. Yoon JH, Kim EK, Kwak JY, et al. Sonographic features and ultrasonography-guided fine-needle aspiration of metastases to the thyroid gland. Ultrasonography 2014;33:40-8

10. Liang $\mathrm{Y}$, Zhang $\mathrm{H}$, Song $\mathrm{X}$, et al. Metastatic heterogeneity of breast cancer: Molecular mechanism and potential therapeutic targets. Semin Cancer Biol 2020;60:14-27.

11. Kiziltan G, Bozdogan N, Ozaslan C. Breast cancer metastasis into thyroid papillary carcinoma: A case report. Breast J 2021;27:547-9.

12. Raveendrannair AK, Mathews A, Varghese BT, et al. Papillary carcinoma thyroid serving as recipient tumor to carcinoma breast: A rare example of tumor-to-tumor metastasis. Indian J Pathol Microbiol 2019;62:122-4.

13. Zhou L, Chen L, Xu D, et al. Breast cancer metastasis to thyroid: a retrospective analysis. Afr Health Sci 2017;17:1035-43

14. Plonczak AM, DiMarco AN, Dina R, et al. Breast cancer metastases to the thyroid gland - an uncommon sentinel for diffuse metastatic disease: a case report and review of the literature. J Med Case Rep 2017;11:269.

15. De la Cruz C, Moriya T, Endoh M, et al. Invasive micropapillary carcinoma of the breast: clinicopathological and immunohistochemical study. Pathol Int 2004;54:90-6.

16. Amendoeira I, Magalhães J, Damasceno M. Invasive micropapillary carcinoma of the breast: are the pure forms more aggressive than the mixed forms? Breast J 2003;9:337-8.

17. Guan X, Xu G, Shi A, et al. Comparison of 
clinicopathological characteristics and prognosis among patients with pure invasive ductal carcinoma, invasive ductal carcinoma coexisted with invasive micropapillary carcinoma, and invasive ductal carcinoma coexisted with ductal carcinoma in situ: A retrospective cohort study. Medicine (Baltimore) 2020;99:e23487.

(English Language Editors: E. Davies and J. Gray)

Cite this article as: Yu Y, Wang R, Deng J, Zhou J, Zhou H, Wang J. Invasive micropapillary carcinoma of mixed breast cancer metastasizing to the cervical region and thyroid: report of a rare case. Gland Surg 2021;10(11):3141-3146. doi: 10.21037/ gs-21-506 


\section{Supplementary}

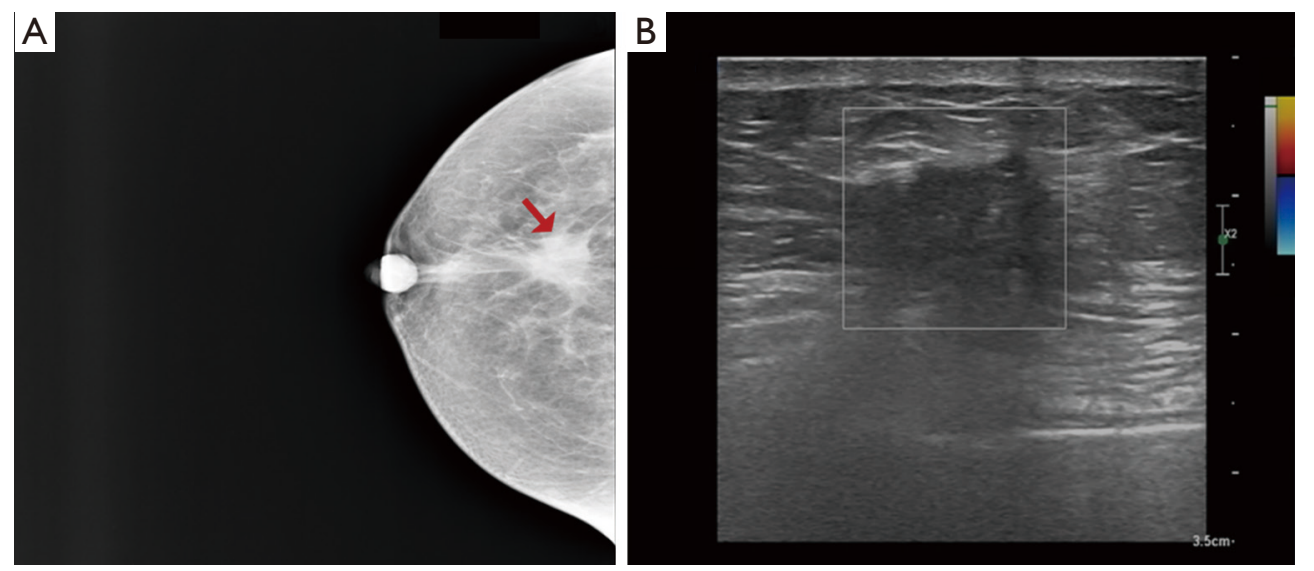

Figure S1 Images of the breast mass. (A) A bilateral mammography showed an irregular hyperdense mass in the right retro areolar region (arrow). (B) Ultrasonography revealed a $1.7 \mathrm{~cm} \times 1.3 \mathrm{~cm}$ hypoechoic, irregular mass with no blood flow. 